\title{
Survey on Ethno Botany and Medicinal Animals at Sayo and Hawa Gelan Districts of Kelem Wollega Zone, Western Ethiopia
}

\author{
Debela Abdeta*, Morka Amante and Yobsan Tamiru \\ Wollega University School of Veterinary Medicine, Ethiopia \\ *Corresponding author: Debela Abdeta, Wollega University School of Veterinary Medicine, Ethiopia
}

ARTICLE INFO

Received: 慧 March 27, 2020

Published:

Citation: Debela A, Morka A, Yobsan T. Survey on Ethno Botany and Medicinal Animals at Sayo and Hawa Gelan Districts of Kelem Wollega Zone, Western Ethiopia. Biomed J Sci \& Tech Res 28(2)-2020. BJSTR. MS.ID.004620.

Keywords: Animals; Ethno Practitioners; Hawa Galan; Human Beings; Plants; Sayo

\author{
ABSTRACT
}

Millions of people around the globe use flora and fauna as source of ethno medicine since ancient times. Despite the fact that ethno medicine has been very crucial for the health of animal and human beings it has not yet been well documented, and much effort is needed in research and integration activities in Ethiopia. It is important to document the traditional knowledge of human communities, since the majority of such communities are losing their socioeconomic and cultural characteristics. This research was focused on assessing and identifying the knowledge of ethno medicine practices used for maintaining the health and curing diseases of humans and livestock in Sayo and Hawa Galan Districts. A purposive Study conducted from January 2018 to June 2019 on Ethno practitioners indicates sixty-six (66) different plant species under 38 families and 10 different animal species are used as medicine for different human and animal ailments. The most common herbal preparation method is concoction with oral route frequently used. Shrubs, herbs and trees are the common habitats whereas leaf, root, seed and coat are common parts of plants used as medicine. Different animal parts like hoof, blood, meat, liver is commonly used with different additives like milk, honey, salt.

This study showed the wide use of medicinal plants and animals in Hawa Galan and Sayo District in meeting the primary healthcare needs of the community. Even though there is a wealth of indigenous knowledge transfer is declining from generation to generation as a result of oral transmission. This finding therefore may imply that conducting in-depth scientific studies may help elucidate the science underlying the efficacy of both animals and plants in managing health and this may lead to the discovery of useful Lead compound. Further studies concerning the conservation and management of the animal and plant resources are needed.

\section{Introduction}

Millions of people around the world have an intimate relationship with their livestock. Ethiopia is the home of many nationalities and remarkably diverse flora and fauna, including numerous endemic species that are utilized in the different traditional medical practices. In Ethiopia, people have been using both plant and animal species for medication of different animal and human diseases over centuries when there was no modern health service delivery [1]. Ethnomedicine is being defined as a mode of identifying, use and integration of the local knowledges, related skills and custom procedures created by people for purpose of preserving health and welfare of working and productive animals [2]. They also studies traditional knowledge, folk beliefs, skills, methods and practices used for the treatment of livestock ailments. There are local healers, who are knowledgeable and experienced in traditional veterinary health care. They use the locally available medicinal plants for treatment of animals and humans. The ethno veterinary systems are ecosystem and ethnic-community specific and therefore, the characteristics, sophistication, and intensity of these systems differ greatly among individuals, societies, and regions. The traditional medical knowledge of indigenous people 
across the globe has played an important role in identifying living organisms which are important for treating Human and livestock health problems [3].

The practice of ethno veterinary medicine has lagged behind that of its counterpart (modern veterinary medicine) many times partly because the practice was secretly done, and its information hidden in the gray literature [4]. The identification and acquisition of this knowledge was no means an easy task in the lives of people. It was a gradual process of trial and error mechanisms, which must have caused many a fatality before coming to its current status [5]. The discovery of those practices must have occurred in a number of ways, not only by the principle of trial and error mechanism. According to the World Health Organization (1993) at least 80\% of people in developing countries depend largely on indigenous practices for the control and treatment of various diseases affecting both human beings and their animals [1]. Of the 252 essential chemicals selected by the World Health Organization, $8.7 \%$ came from animals [6]. Since ancient times, animals and their Products have been used in the preparation of traditional remedies in various cultures [7]. General observations and studies show that the farmers in developing countries are using ethno veterinary practices for curing various diseases of their livestock. Recent revival of Western interest in traditional veterinary medicine followed a revived interest in traditional practices in human health [8]. The importance of traditional medicine as a source of primary health care was first officially recognized by the WHO in the Primary Health Care Declaration of Alma-Ata (1978) and has been globally addressed since 1978 by the Traditional Programme of the WHO. Despite ethno veterinary medicine being the equivalent body of knowledge for livestock that ethno medicine is for humans, it still lacks a high level of institutional backing from the World Organization for Animal Health (OIE) or from FAO [9].

Worldwide interest in documenting and validating ethno vet practices arose in the early 1980s, as people started to realize that ethno vet knowledge was disappearing. Elderly community members with this knowledge were dying and the introduction of modern practices made it difficult for the younger generations to appreciate and use the beliefs and practices of their ancestors. Interest in ethno vet practices has grown recently because these practices are much less prone to drug resistance and have fewer damaging side-effects on the environment than conventional medicine [1]. In many native and local stock raising communities if not all, a considerable proportion of useful ethno knowledge and some of the traditional animal health care practices remain unknown to date, albeit their increased demand to be integrated into primary animal health care delivery systems for wider use by rural and peri-urban communities. The folk health practices largely remain undocumented and are passed on from one generation to the other by word of mouth [9]. The healing of human ailments by using therapeutics-based medicines obtained from animals is called zootherapy [10]. Wild and domestic animals and their by-products such as hooves, skins, bones, feathers, and tusks are important ingredients in the preparation of curative, protective and preventive medicine $[11,12]$.

\section{Research Gap}

Despite the fact that ethno medicine has been very crucial for the animal health care of most developing countries, it has not yet been well documented, and much effort is needed in research and integration activities in these countries. The current loss of medicinal organisms in the country due to natural and anthropogenic factors links with the missing of valuable indigenous knowledge associated with the plants and animals. Most times, it is impossible to document all the knowledge of traditional healers. Hence critical observations of traditional medicine practices of the community should make for the selection of plants that are worth documenting. In many developing countries, medicinal plants have not been well studied, tested or documented. Most of the information is still in the hands of traditional healers and knowledge of healers is either lost or passed to generation by the word of mouth. Thus, ethnobotanical research attempts to document the knowledge of the healers in the community in order to reserve it for future use [13]. Loss of traditional knowledge has impact on the development of modern medicine [14].

\section{Justification}

It is important to document the traditional knowledge of human communities, since the majority of such communities are losing their socioeconomic and cultural characteristics [14]. In Ethiopia, many ethnic communities mainly use local traditional medicine for their health care. Work has been done on ethnobotany with few reports ethnozoology in Ethiopia but there is lacking published documents in Sayo and Hawa Galan districts. Therefore, this research was focused on.

a) Assessing and identifying ethno medicine knowledge and practices used for maintaining the health and curing diseases of humans and livestock

\section{Materials and Methods}

A purposive Study was conducted from January 2018 to June 2019 on Ethnopractitioners offering primary healthcare services to both livestock and humans.

\section{Description of the Study Area}

The study was conducted in Sayo and Hawa Galan district which is located in Kellem Wollega zone, Oromia Regional state, Western Ethiopia. Dembi-Dollo is capital of Kellem Wollega zone and situated about $652 \mathrm{~km}$ West of Addis Ababa. The study site (Sayo district) has a latitude $8^{\circ} 32^{\prime} \mathrm{N}$ and longitude $34^{\circ} 48^{\prime} \mathrm{E}$ with an elevation between 1700 and 1827 meters above sea level. It has 
tropical climate and remains mostly hot and humid throughout the year. The area receives an average annual rainfall of 700 to 1100 $\mathrm{mm}$. The zone is bordered by West Wollega zone to the north, Gambella regional state to the south, Illubabor zone to the east and Benishangul Gumuz regional state to the west. Hawa Galan is one of the woredas in the Oromia Region of Ethiopia bordered on the south and southwest by Sayo, on the north by Yemalogi Welele, on the northeast by Dale Wabera, and on the south and southeast by the Illubabor Zone. The administrative center of this woreda is Rob Gebeya. Hawa Gelan was separated from former Hawa Welele woreda. The 2007 national census reported a total population for this woreda of 95,976, of whom 49,312 were men and 46,664 were women; 5,562 or $5.8 \%$ of its population were urban dwellers. The majority of the inhabitants observed Islam, with $45.43 \%$ reporting that as their religion, while $32.42 \%$ observed Ethiopian Orthodox Christianity, $20.48 \%$ were Protestants, and $1.31 \%$ were Catholic.

\section{Study Methods}

Semi-structured interviewees, observation and guided field walks with informants was employed to obtain ethnobotanical data. For this study purposive sampling was employed to identify potential informants, kebeles and districts. Recommended traditional medicine practitioners was identified as potential informants and subsequently participated in personal interviews. Interviews were based on a checklist of questions prepared before hand in English and translated to the local language (Afan Oromo). Information regarding local names of medicinal plants, preparation methods, part(s) used, diseases treated, dosage used and route of application was recorded at the spot. Observations were made on the morphological features and habitats of each medicinal plant species in the field. The ethnozoological data (local name of animals, mode of preparation and administration, organ of the animal used, and ingredients added if possible) were collected through semistructured interview $[15,16]$ with informants in parallel with medicinal animals.

Before the start of this project, prior informed consent was sought from individual key respondents through the local administration office. Knowledge of ethnoveterinary medicine was surveyed and documented. Local ethnopractitioners/general traditional healers were consulted because each was associated with a specific aspect of ethno knowledge relevant to the study. Fruitful initial contacts were made and more ethnopractitioners were identified using their existing networks. In order to evaluate the reliability of the information gathered, each key respondent was visited at twice on the same idea to prove the validity of the information given out during the first visit before its final documentation [17].

\section{Collection of Specimens of Plants}

Following a personal interview with the selected key respondents, a field trip was made to identify and collect the listed plant specimens. The specimens were harvested, prepared, packaged and stored according to the herbarium rules and regulations until transported to Herbarium at Addis Ababa University for botanical identification using voucher specimens (DA001-DA 066) and according to the Hutchinson system of plant taxonomy based on the plants' probable phylogeny. For each plant species collected from the field, a voucher specimen was prepared and deposited in the national Herbarium Addis Ababa University with voucher number (DA01-DA066).

\section{Data Analysis}

Microsoft Excel spreadsheet software was used to determine the proportions on growth habit, plant part(s) used, condition of medicinal plants, mode of administration and categories of diseases. For medicinal animal's local name of animals, mode of preparation and target patient treated was analyzed and presented in percentage.

\section{Results}

Sixty-six different plant species and ten different animal species were claimed by local healers having medicinal value. From fortyfour (44) respondent's majority of them were males aged between 35-46 years old, with formal education (Table 1). A list of plants and plant products traditionally used to manage animal and human health amongst the Sayo and Hawa Galan district, including their scientific and vernacular names, growth habits, family names, disease and ill-health conditions treated, target type of patient and the preparation forms of different remedies was made (Table 2). The names of plants were arranged according to their alphabetical order. The classification of plant specimens into growth life forms and/ or habits was also made based on the definition and description of Yumoto et al. 1994 [18]. A total of 66 plant species distributed in 38 families were documented to be used in livestock and human health management by traditional healthcare providers in the study area. Growth life forms of the documented plant species was categorized as trees, shrub, and herbs (Tables 3-8). Shrub Constitute the largest category followed by tree and herb respectively. This shows that the most widely used plant habit in the study area is shrub probably due to abundance and easy accessibility [19]. 
Table 1: Background characteristics of sample respondents $(n=44)$.

\begin{tabular}{|c|c|c|c|}
\hline Parameter & & Number & Percentage \\
\hline Sex & Male & 31 & 70.45 \\
\hline & Female & 13 & 29.54 \\
\hline Age & $35-44$ & 12 & 27.27 \\
\hline & $45-60$ & 26 & 59.09 \\
\hline Educational back ground & $>60$ & 6 & 13.63 \\
\hline & Illiterate & 37 & 15.09 \\
\hline
\end{tabular}

Table 2: vernacular name, growth habitat, family name of medicinal plants.

\begin{tabular}{|c|c|c|c|}
\hline Vernacular name & Habitat & Family name & Scientific name and voucher number \\
\hline Abbayyi & Tree & Myrsinaceae & Maisa lanceolata,(DA015) \\
\hline Adaamii & Tree & Euphorbaceae & Euphorbia abbyssinica J.F.Gmel.(DA017) \\
\hline Afarfattuu & Shrub & Dracaenaceae & Dracaena steudneri Engl.,DA(018) \\
\hline Ambabessa & Tree & Fabaceae & Albizia schimperianaOliv(DA01) \\
\hline Ashaangira & Shrub & Solanaceae & Datura stramonium L.(DA03) \\
\hline pappayyaa & Tree & Caricaceae & Carica papaya L.(DA08) \\
\hline Baargamooadii & Tree & Myrtaceae & Eucalyptus globules labing(DA032) \\
\hline Bakkanniisa & Tree & Euphorbiaceae & Croton macrostachyus De(DA051) \\
\hline Barbarre & Shrub & Solanaceae & Capsicum annuum L., (DA033) \\
\hline Bosoqqee & Shrub & Crassulaceae & Kalanchoe lanceolanta V.(DA09) \\
\hline Bunaa & Tree & Rubiaceae & Coffee arabica L.(DA031) \\
\hline Ceeka & Tree & Fabaceae & Calpurnia sub decandra (L'Herit.) Schweick.(DA053) \\
\hline Damakasee & Shrub & Lamiaceae & Ocimum gratissimum L.(DA010) \\
\hline Dhummuga & Tree & Acanthaceae & Justicia schimperiana (Hochst. ex Nees) T. Anders., (DA034) \\
\hline Eebicha & Tree & Asteraeae & Vernonia amygdalina Del.(DA030) \\
\hline Ejersa & Tree & Oleaceae & Olea europaea L. subsp. cuspidata (Wall. ex G. Don) Cif.(DA035) \\
\hline Gambeela & Tree & Rubiaceae & Gardenia ternifolia Schumach. And Thonn.(DA029) \\
\hline Gatama & Tree & Araliaceae & Schefflera volkensii (Engl.) Harms,(DA036) \\
\hline Geeshoo & Tree & Rhamnaceae & Rhamnu sprinoides L' Herit., (DA054) \\
\hline Goommada & Herb & Brassicaceae & Brassica oleracea varcapitata L(DA011) \\
\hline Haanquu & Shrub & Myrsinaceae & Embelia schimperiVatke, (DA055) \\
\hline Hagamsa & Shrub & Apocynaceae & Carissas pinarum L. (DA037) \\
\hline Hancabbii & Shrub & Lamiaceae & Ocimium lamifolium Hochst. Ex. Benth(DA028) \\
\hline Handoodee & Shrub & Phytolaccaceae & Phytolocca dodecandra, L'Hert.(DA038) \\
\hline Hiddafiitii & Shrub & Ranunculaceae & Clematis simensis Fresen.(DA012) \\
\hline Hiddii & Shrub & Solanaceae & Solanum cordatum Forssk, (DA066) \\
\hline Hoomii & Tree & Rocaceae & Prunus Africana (DA013) \\
\hline Ijabuqqee & Shrub & Cucurbitaceae & Cucurbita pepo L. (DA056) \\
\hline Loomii & Tree & Euphorbiaceae & Justicia schimperiana (Nees) T. Anderson(DA057) \\
\hline Maraasisa & Shrub & Lamiaceae & Clerodendrum myricoides (Hochst) Vatke(DA027) \\
\hline Maxxannee & Herb & Amaranthaceae & Achyranthes aspera L.(DA058) \\
\hline Qabarichoo & Shrub & Asteraceae & Echinops kebericho, (DA039) \\
\hline Qararoo & Shrub & Sterculiaceae & Sterculia africana (Lour.)Fiori, (DA59) \\
\hline Anfaarree & Tree & Loganiaceae & Buddleja polystachya Fresen. (DA026) \\
\hline
\end{tabular}




\begin{tabular}{|c|c|c|c|}
\hline Qobboo & Shrub & Euphorbiaceae & Ricinus communis (DA040) \\
\hline Qorichamichii & Tree & Lamiaceae & Ocimum lamiifolium Hochst. exBenth.(DA060) \\
\hline Qullubbiiadii & Herb & Alliaceae & Allium sativum L., (DA041) \\
\hline Shimfaa & Herb & Solanaceae & Lepidium sativum L. (DA061) \\
\hline Sootello & Tree & Fabaceae & Millettiia ferrugineae (Hoscht.) Bak., (DA025) \\
\hline Tamboo & Shrub & Solanaceae & Nicotiana tabacum L. (DA042) \\
\hline Ulmaayii & Shrub & Rutaceae & Clausena anisate (Wild.) Benth (DA062) \\
\hline Waaddeessa & Tree & Boraginaceae & Cordia africana Lam. (DA043) \\
\hline Baal tokkee & Herb & Euphorbiaceae & Acalypha fruticose Forssk., (DA024) \\
\hline Keelloo & Herb & Asteraceae & Bidens macroptera (Sch. Bip. ex Chiov.) Mesfin, (DA044) \\
\hline Kusaaye & Shrub & Verbanaceae & Lippia adoensis var.Adoensis Hochst. Ex Walp.,(DA063) \\
\hline Dheertuu & Shrub & Viscaceae & Viscum tuberculatum A.Rich. (DA023) \\
\hline Fossoo/faca'aa & Shrub & Cucurbitaceae & Curcumis ficifolius A. Rich. (DA064) \\
\hline Heennaa & Tree & Fabaceae & Ndigo feraarrecta Hochst. ex A.Rich, (DA065) \\
\hline Timijjii & Herb & Polygonaceae & Rumex nepalensis Spreng. (DA016) \\
\hline Ulaagaa & Shrub & Boraginaceae & Ehretia cymose Thonn., (DA045) \\
\hline Umbawoo & Shrub & Cucurbitaceae & Momordica foetida Schumach., (DA022) \\
\hline Yeeroo & Shrub & Lamiaceae & Pycnostachys abyssinica Fresen. (DA046) \\
\hline Gurgubbe & Shrub & Urticaceae & Girardinia bullosa (Steud.) Wedd. (DA050) \\
\hline Harangamaa & Shrub & Capparidaceae & Capparis tomentosa Lam. (DA049) \\
\hline Hiddii waraabessa & Shrub & Solanaceae & Solanum marginatum L. f.(DA021) \\
\hline Maangoo & Tree & Anacardiaceae & Mangifera indica L.(DA047) \\
\hline Margaurgaa & Herb & Poaceae & Panicum monticola Hook .f.(DA052) \\
\hline Reejiijaldeessaa & Shrub & Asteraceae & Vernonia auriculifera Hiern.(DA048) \\
\hline Sooyyamqallee & Shrub & Asteraceae & Vernonia adoensisSch. bip. exWalp.(020) \\
\hline Takkadha'aa & Herb & Rubiaceae & Hymenodictyon floribundum (Hochst. \& Steud.) Robinson. (DA019) \\
\hline
\end{tabular}

Table 3: Growth Habitats of claimed traditional plants $(n=66)$.

\begin{tabular}{|c|c|c|c|}
\hline No & Habitat & Number & Percentage \\
\hline 1 & Herb & 10 & 15.1 \\
\hline 2 & Shrub & 30 & 45.5 \\
\hline 3 & Tree & 26 & 39.4 \\
\hline
\end{tabular}

Table 4: Diversity of medicinal plant species belonging to each plant family $(n=38)$.

\begin{tabular}{|c|c|c|c|}
\hline S.NO & Family name & Frequency & Percentage \\
\hline 1 & Acanthaceae & 1 & 2.63 \\
\hline 2 & Alliaceae & 1 & 2.63 \\
\hline 3 & Amaranthaceae & 1 & 2.63 \\
\hline 4 & Anacardiaceae & 1 & 2.63 \\
\hline 5 & Apocynaceae & 1 & 2.63 \\
\hline 6 & Araliaceae & 1 & 2.63 \\
\hline 7 & Asteraceae & 5 & 2.63 \\
\hline 10 & Boraginaceae & 1 & 5.26 \\
\hline 11 & Boraginaceae & 2 & 2.63 \\
\hline 12 & Brassicaceae & 1 & 5.26 \\
\hline
\end{tabular}




\begin{tabular}{|c|c|c|c|}
\hline 13 & Crassulaceae & 1 & 2.63 \\
\hline 14 & Cucurbitaceae & 3 & 7.89 \\
\hline 15 & Dracaenaceae & 1 & 2.63 \\
\hline 16 & Euphorbiaceae & 5 & 13.16 \\
\hline 17 & Fabaceae & 5 & 13.16 \\
\hline 18 & Lamiaceae & 5 & 13.16 \\
\hline 19 & Loganiaceae & 1 & 2.63 \\
\hline 20 & Myrsinaceae & 2 & 5.26 \\
\hline 21 & Myrtaceae & 1 & 2.63 \\
\hline 22 & Oleaceae & 1 & 2.63 \\
\hline 23 & Phytolaccaceae & 1 & 2.63 \\
\hline 24 & Poaceae & 1 & 2.63 \\
\hline 25 & Polygonaceae & 1 & 2.63 \\
\hline 26 & Ranunculaceae & 1 & 2.63 \\
\hline 27 & Rhamnaceae & 1 & 2.63 \\
\hline 28 & Rocaceae & 1 & 2.63 \\
\hline 29 & Rubiaceae & 3 & 7.89 \\
\hline 30 & Rutaceae & 2 & 5.26 \\
\hline 31 & Solanaceae & 6 & 15.78 \\
\hline 32 & Sterculiaceae & 1 & 2.63 \\
\hline 33 & Urticaceae & 1 & 2.63 \\
\hline 34 & Verbanaceae & 1 & 2.63 \\
\hline 35 & Viscaceae & 1 & 2.63 \\
\hline 36 & Melianthaceae & 1 & 2.63 \\
\hline 37 & Simarobouceae & 1 & 2.63 \\
\hline 38 & Zingibiraceae & 1 & 2.63 \\
\hline 39 & Total & & 100.0 \\
\hline
\end{tabular}

Table 5: Medicinal plant parts used in traditional medicines $(\mathrm{N}=87)$.

\begin{tabular}{|c|c|c|c|}
\hline S.no & Parts used & Frequency & Percentage \\
\hline 1 & Leaf & 50 & 57.47 \\
\hline 2 & Seed/fruits & 10 & 11.49 \\
\hline 3 & Root & 15 & 17.24 \\
\hline 4 & Whole plat & 1 & 1.15 \\
\hline 5 & Coat & 11 & 12.64 \\
\hline
\end{tabular}

Table 6: List of medicinal plants with disease they are used to treat.

\begin{tabular}{|c|c|c|c|c|c|c|}
\hline No & Local name & Species name & $\begin{array}{c}\text { Part of plant } \\
\text { used }\end{array}$ & $\begin{array}{c}\text { Disease/ill-health } \\
\text { condition treated }\end{array}$ & $\begin{array}{c}\text { Methods of } \\
\text { preparation }\end{array}$ & Target patient \\
\hline 1 & Abbayyi & Maisa lanceolata, & Leaf & Warts & Squeezing & Human being \\
\hline 2 & Adaamii & $\begin{array}{c}\text { Euphorbia abbyssinica } \\
\text { J.F.Gmel. }\end{array}$ & Bleed & External parasite & Topical & Both \\
\hline 3 & Afarfattuu & Dracaena steudneri Engl., & Leaf & Retained placenta & Concoction & Cattles \\
\hline 4 & Ambabessa & Albizia schimperiana Oliv & Coat & Diarhoea & Concoction & Animals \\
\hline 5 & Ashaangira & Datura stramonium L. & Leed & Evil eye & Concoction & Concoction \\
\hline 6 & Pappayyaa & Carica papaya L. & Leaf & Malaria & Concoction & Human being \\
\hline 7 & Baargamooadii & Eucalyptus globules labing & Leaf & Cough & Smelling & Human being \\
\hline
\end{tabular}




\begin{tabular}{|c|c|c|c|c|c|c|}
\hline 8 & Bakkanniisa & Croton macrostachyus De & $\begin{array}{l}\text { Leaf } \\
\text { Coat } \\
\text { Coat } \\
\text { Coat }\end{array}$ & $\begin{array}{l}\text { Bloat } \\
\text { Internal parasite } \\
\text { Typhoid } \\
\text { Liver and lung diseases }\end{array}$ & $\begin{array}{l}\begin{array}{c}\text { Swallowing the } \\
\text { leaf }\end{array} \\
\text { Eating the grinded } \\
\text { coat } \\
\text { Firing and smell- } \\
\text { ing the smoke } \\
\text { Concoction }\end{array}$ & $\begin{array}{l}\text { Animal } \\
\text { Human being } \\
\text { Animal } \\
\text { Both }\end{array}$ \\
\hline 9 & Barbarre & Capsicum annuum L., & Seed & Internal parasite & Concoction & Human being \\
\hline 10 & Bosoqqee & Kalanchoe lanceolanta V. & $\begin{array}{c}\text { Coat } \\
\text { Whole plant }\end{array}$ & $\begin{array}{c}\text { Snake bite } \\
\text { Aborting agent }\end{array}$ & $\begin{array}{l}\text { Concoction } \\
\text { Concoction }\end{array}$ & $\begin{array}{c}\text { Both } \\
\text { Human being }\end{array}$ \\
\hline 11 & Bunaa & Coffee arabica $\mathrm{L}$ & Leaf & $\begin{array}{c}\text { Malaria or acute dis- } \\
\text { eases }\end{array}$ & $\begin{array}{c}\text { Drinking after } \\
\text { boiling with sugar }\end{array}$ & Human being \\
\hline 12 & Ceeka & $\begin{array}{l}\text { Calpurnia subdecandra } \\
\text { (L'Herit.)Schweick. }\end{array}$ & $\begin{array}{l}\text { Leaf } \\
\text { Leaf }\end{array}$ & $\begin{array}{l}\text { External parasite } \\
\text { Internal parasite }\end{array}$ & $\begin{array}{l}\text { Concoction } \\
\text { Concoction }\end{array}$ & $\begin{array}{l}\text { Animal } \\
\text { Both }\end{array}$ \\
\hline 13 & Damakasee & Ocimum gratissimum L. & $\begin{array}{l}\text { Leaf } \\
\text { Leaf }\end{array}$ & $\begin{array}{l}\text { Common cold } \\
\text { Wound }\end{array}$ & $\begin{array}{c}\text { Boiling and } \\
\text { drinking with tea/ } \\
\text { coffee } \\
\text { Tying with } \\
\text { squeezed leaf }\end{array}$ & Human being \\
\hline 14 & Dhummuga & $\begin{array}{c}\text { Justicia schimperiana } \\
\text { (Hochst. ex Nees) T.An- } \\
\text { ders., }\end{array}$ & $\begin{array}{c}\text { Coat } \\
\text { Root Leaf }\end{array}$ & $\begin{array}{c}\text { Rabies } \\
\text { Rabies } \\
\text { Sneezing and acute } \\
\text { death of poultry }\end{array}$ & $\begin{array}{l}\text { Concoction } \\
\text { Concoction } \\
\text { Concoction }\end{array}$ & $\begin{array}{l}\text { Both } \\
\text { Both } \\
\text { Both }\end{array}$ \\
\hline 15 & Eebicha & Vernonia amygdalina Del. & Leaf & Diarrhea & Concoction & Both \\
\hline 16 & Ejersa & $\begin{array}{l}\text { Olea europaea L. subsp. } \\
\text { cuspidata (Wall. ex G. } \\
\text { Don) Cif. }\end{array}$ & Leaf & Bleeding & $\begin{array}{l}\text { Topical adminis- } \\
\text { tration }\end{array}$ & Both \\
\hline 17 & Gambeela & $\begin{array}{c}\text { Gardenia ternifolia Schum- } \\
\text { ach. And Thonn. }\end{array}$ & Coat & Tonsil & Swallowing & Human being \\
\hline 18 & Gatama & $\begin{array}{c}\text { Schefflera volkensii (Engl.) } \\
\text { Harms, }\end{array}$ & Coat & Trypanosoma & $\begin{array}{l}\text { Drinking Crushed } \\
\text { coat }\end{array}$ & Animal \\
\hline 19 & Geeshoo & $\begin{array}{c}\text { Rhamnus prinoides L' } \\
\text { Herit. }\end{array}$ & Seed & Ring worm & $\begin{array}{l}\text { Adding the grind- } \\
\text { ed powder }\end{array}$ & Both \\
\hline 20 & Goommada & $\begin{array}{c}\text { Brassica oleracea varcap- } \\
\text { itata } \mathrm{L}\end{array}$ & Seed & Trypanosoma & Chewing the seed & Animal \\
\hline 21 & Haanquu & Embelia schimperi Vatke, & $\begin{array}{l}\text { Leaf } \\
\text { Seed }\end{array}$ & $\begin{array}{c}\text { Tick } \\
\text { Taenea }\end{array}$ & $\begin{array}{l}\text { Anointing } \\
\text { Chewing }\end{array}$ & $\begin{array}{c}\text { Animal } \\
\text { Human being }\end{array}$ \\
\hline 22 & Hagamsa & Carissa spinarum L. & Seed & Internal parasite & Concoction & Human being \\
\hline 23 & Hancabbii & $\begin{array}{l}\text { Ocimium lamifolium } \\
\text { Hochst. Ex. Benth }\end{array}$ & Leaf & Acute disease & Concoction & Both \\
\hline 24 & Handoodee & $\begin{array}{c}\text { Phytolocca dodecandra, } \\
\text { L'Hert. }\end{array}$ & $\begin{array}{l}\text { Fruit } \\
\text { Root } \\
\text { Leaf }\end{array}$ & $\begin{array}{c}\text { Rabies } \\
\text { Rabies } \\
\text { Eye lid prolapse }\end{array}$ & $\begin{array}{l}\text { Concoction } \\
\text { Concoction } \\
\text { Squeezing juice }\end{array}$ & $\begin{array}{c}\text { Both } \\
\text { Both } \\
\text { Animals }\end{array}$ \\
\hline 25 & Hiddafiitii & Clematis simensis Fresen. & Leaf & Fasciola & Concoction & Animals \\
\hline 26 & Hiddii & Solanum cordatum Forssk, & Leaf & $\begin{array}{l}\text { Nasal discharge } \\
\text { Bleeding }\end{array}$ & $\begin{array}{l}\text { Concoction } \\
\text { Concoction }\end{array}$ & $\begin{array}{l}\text { Animal } \\
\text { Animal }\end{array}$ \\
\hline 27 & Hoomii & Prunus africana & Coat & Wound & $\begin{array}{l}\text { Adding crushed } \\
\text { powder }\end{array}$ & Both \\
\hline
\end{tabular}




\begin{tabular}{|c|c|c|c|c|c|c|}
\hline 28 & $\begin{array}{l}\text { Buqqee/ } \\
\text { Dabaaqula }\end{array}$ & Cucurbita pepo L. & Seed & Rabies & $\begin{array}{l}\text { Eating the roasted } \\
\text { seed for pregnant } \\
\text { mothers }\end{array}$ & Human being \\
\hline 29 & Loomii & $\begin{array}{l}\text { Justicia schimperiana } \\
\text { (Nees) T. Anderson }\end{array}$ & Fruit & Snake poisoning & $\begin{array}{l}\text { Drinking the } \\
\text { squeezed Juice }\end{array}$ & Both \\
\hline 30 & Maraasisa & $\begin{array}{l}\text { Clerodendrum myricoides } \\
\text { (Hochst) Vatke }\end{array}$ & Root & Stomach pain & Chewing & Animal \\
\hline 31 & Maxxannee & Achyranthes aspera L. & Leaf & Wound & Concoction & Animal \\
\hline 32 & Qabarichoo & $\begin{array}{c}\text { Echinops kebericho, } \\
\text { Mesfin }\end{array}$ & Root & $\begin{array}{c}\text { Typhoid } \\
\text { White worm } \\
\text { Stomach pain } \\
\text { Evil eye } \\
\text { Diarrhea }\end{array}$ & $\begin{array}{l}\text { Concoction } \\
\text { Concoction } \\
\text { Chewing } \\
\text { Smoke } \\
\text { Concoction }\end{array}$ & $\begin{array}{c}\text { Human being } \\
\text { Animal } \\
\text { Human being } \\
\text { Human being } \\
\text { Animal }\end{array}$ \\
\hline 33 & Qararoo & $\begin{array}{c}\text { Sterculia africana (Lour.) } \\
\text { Fiori, }\end{array}$ & Root & $\begin{array}{c}\text { Male sexual impotence } \\
\text { Diarhoea }\end{array}$ & $\begin{array}{l}\text { Concoction } \\
\text { Concoction }\end{array}$ & $\begin{array}{l}\text { Human being } \\
\text { Human being }\end{array}$ \\
\hline 34 & Anfaarree & $\begin{array}{l}\text { Buddle japolystachya } \\
\text { Fresen. }\end{array}$ & Leaf & Eye disease & Squeezing & Animal \\
\hline 35 & Qobboo & Ricinus communis & Leaf & Retained fetal placenta & Concoction & Animal \\
\hline 36 & $\begin{array}{c}\text { Qorichamichii/ } \\
\text { Qasee }\end{array}$ & $\begin{array}{l}\text { Ocimum lamiifolium } \\
\text { Hochst. exBenth. }\end{array}$ & Leaf & Michii & $\begin{array}{l}\text { Warming the leaf } \\
\text { and burning the } \\
\text { face }\end{array}$ & Animal \\
\hline 37 & Qullubbiiadii & Allium sativum L., & $\begin{array}{l}\text { Root } \\
\text { Root }\end{array}$ & $\begin{array}{l}\text { Stomach pain } \\
\text { Tooth pain }\end{array}$ & $\begin{array}{c}\text { Swallowing } \\
\text { Chewing }\end{array}$ & $\begin{array}{c}\text { Both } \\
\text { Human being }\end{array}$ \\
\hline 38 & Shimfaa & Lepidium sativum $\mathrm{L}$. & $\begin{array}{l}\text { Seed } \\
\text { Seed } \\
\text { Leaf }\end{array}$ & $\begin{array}{l}\text { Trypanosomiasis } \\
\text { Bloat } \\
\text { Trypanosomiasis }\end{array}$ & $\begin{array}{l}\text { Concoction } \\
\text { Concoction } \\
\text { Concoction }\end{array}$ & Animal \\
\hline 39 & Sootello & $\begin{array}{l}\text { Millettii aferrugineae } \\
\text { (Hoscht.) Bak., }\end{array}$ & Coat & Bleeding & $\begin{array}{l}\text { Tying to bleeding } \\
\text { area }\end{array}$ & Animal \\
\hline 40 & Tamboo & Nicotiana tabacum L. & $\begin{array}{l}\text { Leaf } \\
\text { Leaf }\end{array}$ & $\begin{array}{l}\text { Tick and Leeches } \\
\text { Snake repellent }\end{array}$ & $\begin{array}{l}\text { Topical and intra- } \\
\text { nasal application } \\
\text { of squeezed plant } \\
\text { Smoke }\end{array}$ & $\begin{array}{l}\text { Animal } \\
\text { Both }\end{array}$ \\
\hline 41 & Ulmaayii & $\begin{array}{c}\text { Clausena anisate (Wild.) } \\
\text { Benth }\end{array}$ & Leaf & Fleas and lice & $\begin{array}{l}\text { Spreading in the } \\
\text { room }\end{array}$ & Both \\
\hline 42 & Waaddeessa & Cordia africana Lam. & $\begin{array}{l}\text { Leaf } \\
\text { Coat }\end{array}$ & $\begin{array}{l}\text { Spider toxin/urine } \\
\text { Bleeding }\end{array}$ & $\begin{array}{l}\text { Squeezing the } \\
\text { juice }\end{array}$ & $\begin{array}{c}\text { Human being } \\
\text { Animal }\end{array}$ \\
\hline 43 & Baal tokkee & Acalypha fruticose Forssk., & Leaf & Fattening & Eating & Animal \\
\hline 44 & Keelloo & $\begin{array}{l}\text { Bidens macroptera (Sch. } \\
\text { Bip. ex Chiov.) Mesfin, }\end{array}$ & Leaf & Bleeding & $\begin{array}{l}\text { Anointing with } \\
\text { juice }\end{array}$ & Human being \\
\hline 45 & Kusaaye & $\begin{array}{c}\text { Lippia adoensis var. } \\
\text { Adoensis Hochst. Ex Walp., }\end{array}$ & Leaf & Common cold & Smelling fresh leaf & Human being \\
\hline 46 & Dheertuu & $\begin{array}{c}\text { Viscum tuberculatum } \\
\text { A.Rich. }\end{array}$ & Leaf & $\begin{array}{c}\text { Malaria } \\
\text { Acute disease }\end{array}$ & $\begin{array}{l}\text { Drinking after } \\
\text { Boiling with sugar } \\
\text { Concoction }\end{array}$ & $\begin{array}{c}\text { Human being } \\
\text { Animal }\end{array}$ \\
\hline
\end{tabular}




\begin{tabular}{|c|c|c|c|c|c|c|}
\hline 47 & Fossoo/faca'aa & Curcumis ficifolius A. Rich. & Leaf & $\begin{array}{c}\text { Bloat } \\
\text { Internal worm } \\
\text { Blood clot under tongue }\end{array}$ & $\begin{array}{l}\text { Concoction } \\
\text { Concoction } \\
\text { Concoction }\end{array}$ & $\begin{array}{l}\text { Animal } \\
\text { Animal } \\
\text { Animal }\end{array}$ \\
\hline 48 & Heennaa & $\begin{array}{l}\text { Ndigofera arrecta Hochst. } \\
\text { ex A.Rich, }\end{array}$ & $\begin{array}{l}\text { Leaf } \\
\text { Leaf }\end{array}$ & $\begin{array}{l}\text { Malaria } \\
\text { Equine colic }\end{array}$ & $\begin{array}{l}\text { Drinking boiled } \\
\text { leaf with sugar } \\
\text { Concoction }\end{array}$ & $\begin{array}{c}\text { Man } \\
\text { Equine }\end{array}$ \\
\hline 49 & Timijjii & Rumex nepalensis Spreng. & Leaf & Stomach pain & Drinking juice & Both \\
\hline 50 & Ulaagaa & Ehretia cymosa Thonn., & $\begin{array}{l}\text { Leaf } \\
\text { Leaf }\end{array}$ & $\begin{array}{l}\text { Warts } \\
\text { Wound }\end{array}$ & $\begin{array}{c}\text { Tying with leaf } \\
\text { Concoction }\end{array}$ & $\begin{array}{l}\text { Both } \\
\text { Both }\end{array}$ \\
\hline 51 & Umbawoo & $\begin{array}{l}\text { Momordica foetida } \\
\text { Schumach. }\end{array}$ & $\begin{array}{l}\text { Fruits } \\
\text { Fruits }\end{array}$ & $\begin{array}{c}\text { External Parasite } \\
\text { Warts }\end{array}$ & $\begin{array}{l}\text { Concoction } \\
\text { Lotion/cream }\end{array}$ & $\begin{array}{l}\text { Animal } \\
\text { Both }\end{array}$ \\
\hline 52 & Yeeroo & $\begin{array}{l}\text { Pycnostachys abyssinica } \\
\text { Fresen. }\end{array}$ & Leaf & Rabies & Concoction & Both \\
\hline 53 & Gurgubbe & $\begin{array}{l}\text { Girardinia bullosa (Steud.) } \\
\text { Wedd. }\end{array}$ & $\begin{array}{l}\text { Leaf } \\
\text { Root }\end{array}$ & $\begin{array}{c}\text { Lice and fleas } \\
\text { Internal parasite }\end{array}$ & $\begin{array}{l}\text { Concoction } \\
\text { Concoction }\end{array}$ & $\begin{array}{l}\text { Animals } \\
\text { Animals }\end{array}$ \\
\hline 54 & $\begin{array}{l}\text { Harangamagur- } \\
\text { racha }\end{array}$ & Capparis tomentosa Lam. & Root & Tooth pain & $\begin{array}{l}\text { Swallowing the } \\
\text { crushed root }\end{array}$ & Human being \\
\hline 55 & $\begin{array}{c}\text { Hiddii } \\
\text { waraabessa }\end{array}$ & Solanum marginatum L.f. & $\begin{array}{l}\text { Seed } \\
\text { Seed }\end{array}$ & $\begin{array}{l}\text { Nasal bot in sheep } \\
\text { Tooth pain }\end{array}$ & $\begin{array}{l}\text { Concoction } \\
\text { Chewing }\end{array}$ & $\begin{array}{l}\text { Sheep } \\
\text { Both }\end{array}$ \\
\hline 56 & Maangoo & Mangifera indica L. & Leaf & Fever & $\begin{array}{c}\text { Drinking after } \\
\text { boiling with sugar }\end{array}$ & Human being \\
\hline 57 & Marga urgaa & $\begin{array}{c}\text { Panicum monticola } \\
\text { Hook.f. }\end{array}$ & $\begin{array}{l}\text { Leaf } \\
\text { Leaf }\end{array}$ & $\begin{array}{l}\text { Vomiting } \\
\text { Evil eye }\end{array}$ & $\begin{array}{l}\text { Smelling leaf } \\
\text { Spreading in/ } \\
\text { around the home }\end{array}$ & $\begin{array}{c}\text { Human being } \\
\text { Both }\end{array}$ \\
\hline 58 & $\begin{array}{l}\text { Reejjii jaldees- } \\
\text { saa }\end{array}$ & $\begin{array}{c}\text { Vernonia auriculifera } \\
\text { Hiern. }\end{array}$ & Leaf & Black leg & Concoction & Animals \\
\hline 59 & Sooyyam qallee & $\begin{array}{l}\text { Vernonia adoensis Sch. } \\
\text { bip. exWalp. }\end{array}$ & Leaf & Acute disease & Smoke & Animals \\
\hline 60 & Takkadha'aa & $\begin{array}{l}\text { Hymenodictyon floribun- } \\
\text { dum (Hochst. \&Steud.) } \\
\text { Robinson. }\end{array}$ & Leaf & Black leg & Concoction & Animals \\
\hline 61 & Harbuu & Ritchieal bersii Gilg & Fruit & Wound & $\begin{array}{l}\text { Drops from stem } \\
\text { tied on the } \\
\text { wound }\end{array}$ & Animals \\
\hline 62 & Laaftoo & $\begin{array}{l}\text { Acacia abyssinica } \\
\text { Hochst ex.Benth. }\end{array}$ & Leaf & Goiter & $\begin{array}{l}\text { Leaf of A. abyssini- } \\
\text { ca is smashed } \\
\text { and the sap is } \\
\text { made }\end{array}$ & Human being \\
\hline 63 & Lolchiisaa & $\begin{array}{c}\text { Bersama abyssinica } \\
\text { Fresen }\end{array}$ & Leaf & Wound & $\begin{array}{l}\text { Leafy-Stem is } \\
\text { squeezed and } \\
\text { creamed on } \\
\text { wound }\end{array}$ & Human being \\
\hline 64 & Cilaattama & Ruta chalepensis L. & Leaf & Stomach pain & $\begin{array}{c}\text { Leaf is } \\
\text { smashed } \\
\text { one cup of do- } \\
\text { mestic } \\
\text { alcohol is taken by } \\
\text { human }\end{array}$ & Human being \\
\hline
\end{tabular}




\begin{tabular}{|c|c|c|c|c|c|c|}
\hline 65 & Jinjibla & $\begin{array}{c}\text { Zingiber } \\
\text { officinale Roscoe }\end{array}$ & Root & Influenza & $\begin{array}{l}\text { Pounded with } \\
\text { other and drink } \\
\text { after boiling }\end{array}$ & Human being \\
\hline 66 & Qomonyoo & $\begin{array}{c}\text { Brucea } \\
\text { antidysentrica Fresen }\end{array}$ & $\begin{array}{l}\text { Leaf } \\
\text { Root }\end{array}$ & $\begin{array}{c}\text { External parasite } \\
\text { Rabies }\end{array}$ & $\begin{array}{l}\text { pounded Leaf } \\
\text { mixed with water } \\
\text { is anointed } \\
\text { crushed and } \\
\text { watered }\end{array}$ & $\begin{array}{c}\text { Human being } \\
\text { Both }\end{array}$ \\
\hline
\end{tabular}

NB: acute disease indicates a syndrome called 'michii' in 'Afaan Oromo'

Table 7: Methods used in preparation of herbal remedies $(\mathrm{N}=84)$.

\begin{tabular}{|c|c|c|c|}
\hline No. & Methods of preparation & Frequency & Percentage \\
\hline 1 & Squeezing & 9 & 52.71 \\
\hline 2 & Concoction & 44 & 5.95 \\
\hline 3 & Smoking/Smelling & 5 & 7.14 \\
\hline 4 & Boiling & 3 & 3.57 \\
\hline 5 & Tying & 3 & 3.57 \\
\hline 6 & Anointing & 11 & 13.1 \\
\hline 7 & Eating/chewing & 2 & 2.38 \\
\hline 9 & Adding grinded powder & 84 & 100.0 \\
\hline
\end{tabular}

Table 8: Roots of administration of traditional herbal medicine $(n=100)$.

\begin{tabular}{|c|c|c|}
\hline Parts used & Number & Percentage \\
\hline Oral & 55 & 55.0 \\
\hline Topical & 32 & 32.0 \\
\hline Intranasal & 10 & 10.0 \\
\hline Intraocular & 3 & 3.0 \\
\hline
\end{tabular}

Table 9: List of medicinal animals, diseases treated, and parts of animals used in the study area.

\begin{tabular}{|c|c|c|c|c|c|c|}
\hline S.NO & $\begin{array}{l}\text { Vernacular } \\
\text { name }\end{array}$ & Species name & Family & Diseases treated & Parts used & Condition of animal used \\
\hline 1 & Harree & Aquus asinus & Equidae & Typhoid & Nail & Smoking of the nail \\
\hline 2 & Karkarroo & $\begin{array}{l}\text { Phacochoerus } \\
\text { africanus }\end{array}$ & Suidae & Many disease & $\begin{array}{l}\text { Liver } \\
\text { Urine }\end{array}$ & $\begin{array}{l}\text { Eating of fresh liver } \\
\text { Drinking the urine }\end{array}$ \\
\hline 3 & Daamuu & Apis dorsata & Apidae & Cough & Honey & Eating of two spoons in the morning \\
\hline 4 & Xaddee & Hystrix & & $\begin{array}{c}\text { Hepatitis } \\
\text { Fattening } \\
\text { Heart failure }\end{array}$ & $\begin{array}{l}\text { Liver, lung, meat } \\
\text { Liver, Lung, Meat }\end{array}$ & $\begin{array}{c}\text { Eating fresh organs } \\
\text { Eating the soup of organs } \\
\text { Eating organs } \\
\text { Eating organs }\end{array}$ \\
\hline 5 & Booyyee & $\begin{array}{l}\text { Susscrofa do- } \\
\text { mesticus }\end{array}$ & & Trypanosoma & Blood & Drinking fresh blood \\
\hline 6 & Oslee & $\begin{array}{l}\text { Procavia cap- } \\
\quad \text { ensis }\end{array}$ & & Fattening & Meat & Eating the meat \\
\hline 7 & Buutii & Python molorus & & $\begin{array}{l}\text { Python molorus } \\
\text { bite }\end{array}$ & Whole organism & $\begin{array}{l}\text { Killing and smoking for Python } \\
\text { molorus bite }\end{array}$ \\
\hline 8 & Hen & $\begin{array}{l}\text { Gallus gallus } \\
\text { domesticus }\end{array}$ & & Diarhoea & Liver & Eating uncooked liver \\
\hline 9 & Bat & Chiroptera & & Bat disease & Whole organism & Tying the died dried bat to the wound \\
\hline 10 & Cow & Bos taurus & Bovidae & Snake poisoning & Feces & Drinking fresh feces with ash \\
\hline
\end{tabular}


The local names and associated attributes of medicinal animals were recorded for each of the species. In the study area, different parts or products of animals were used to treat different types of ailments. This study revealed the traditional medicinal knowledge of treating various kinds of ailments using different animals and their parts/products by local inhabitants of Sayo and Hawa Galan districts. In the present study, ten (10) species of medicinal animals were collected for treating different ailments of human and animals (Table 9).

\section{Discussion}

The results of the present study showed that Hawa Galan and Sayo districts have a high diversity of medicinal plants and animals useful to treat the people and animals living in the area. Despite the gradual socio-cultural transformation, the inhabitants have retained remarkable knowledge of the medicinal plants and animals with their uses. Ethnoknowledge was transmitted orally and secretly within family lineages. These findings are in agreement with numerous other studies previously carried out in northern Ethiopia and Brasil $[20,21]$. This study revealed that the family as a unit is still a major source of ethnoknowledge for healing, training and gaining experience for many medical ethnopractitioners, whether for humans or animals. In regard to the part(s) of plants harvested and used in ethnomedicine leaf accounting for $57.47 \%$ followed by coat and root which accounts $17.24 \%$ and 12.64 respectively. These results are in an agreement with the previous findings of Guluma et al, 2017, who conducted a similar survey Mida Kegn, West Showa. Leaves from plants therefore appear to be the most preferred harvested parts of plants by ethnopractitioners for us in ethnomedicines [22]. Reports from Horro Guduru Wollega of Ethiopia indicate that Roots and leaves are involved in most of plant induced intoxication [23]. Putting into consideration the biological function of the leaves on plants, the method of harvesting medicinal plants by picking leaves can be very devastating and a threat to the survival of the target plant, more particularly, if the young tender leaves are harvested instead of the old ones, which are almost dropping off the plant to become humus. Similarly, frequent harvesting of roots and barks, the second most preferred parts of plants, may be destructive and unsustainable, thus risking the extinction of the target plant species, and is therefore not advisable.

Ethnoveterinarians in the study area employed a number of methods for preparing herbal remedies. These methods largely depended on the type of the plant used, parts of plants employed, type of disease/ill-health condition and the animal species being treated. Some of the most frequently used methods of ethnoformulation preparations in the study area include concoction, anointing, fumigation and spreading in the home. The survey study further found out that most of the remedies were prepared from a single plant species but treating rabies for both animal and human being involves three or more plants which includes Brucea antidysentrica Fresen, Pycnostachys abyssinica
Fresen. Phytolocca dodecandra, L'Hert., Justicia schimperiana (Hochst. ex Nees) T.Anders with different additives like milk and water. Other prominent preparations however, involved the mixtures of different plant species and at times addition of one or more nonplant ingredients or additives such as milk, soup, honey, porridge, animal fat, salt etc. The use of more than one plant to make ethnoformulations are commonly used in the study area and respondents believed that such an ethnoformulation conferred some synergistic effects to the herbal remedies in certain cases where ingredients of two or more plants were considered to be more effective against a particular disease/ill-health condition than the use of individual plants separately.

On other hand, the use of more than one plant to make ethnoformulations was believed to neutralize toxicity effects and/or bitterness of one part of the ethnoformulation preparation to make it palatable and easily administered. While making the remedial preparations from plants and plant products, the most frequently used solvent was water, particularly during the preparations of concoctions and juices with the addition other additives (milk, honey, animal fat and salt). This point of view depends largely on one's ethnicity and cultural belief and taboos [24]. An accurate knowledge about the symptoms, signs through audio and visual preceded the choice of an appropriate treatment and management strategies.

Cattle, sheep, equine, poultry and human beings are frequently treated by traditional medicine. The number of known ethnoveterinary remedies for a particular type of livestock may probably correspond with socio-economic value and importance of the animal in their cultural and traditional life and perhaps this may also explain the order of acquisition of these animals for domestication by that people [25]. The route of administration of ethnobotanical preparations depended on the nature of the disease and the target patient [26]. The main routes of administration documented in the study area were oral $55 \%$, topical $32 \%$ and intranasal $10 \%$. Application of ethnomedicines through the eyes and other routes of administration were also practiced. Correct dosage (as described by an ethnopractitioner such as three glasses in a day) was an important aspect of ethnoveterinary medicine according to the respondents because, under dose was known to make the remedy ineffective while overdose caused livestock poisoning and subsequent death. Many respondents were of the opinion that the correct dosages for various ethnomedicines had been established through a lengthy period of trial and error mechanisms.

In this study, 10 animal species or their products were identified and believed to cure different ailments. Other studies reported in Ethiopia showed that approximately 23 animals and/or their parts were identified to be used in traditional medicines in Degu tribes in Tigray region [27]. Sixteen species of medicinal animals were collected and identified for treating 18 different human ailments in the Kafta-Humera District, Northern Ethiopia [28]. The study 
conducted by Borah and Prasad recorded a total of 44 different species of animals which are used for the treatments of 40 different ailments [29]. Information regarding the way to acquire traditional medicinal knowledge, duration of time to use traditional medicine, the reason that forces the people to use traditional medicines, categories of people that use traditional medicine, the outlooks of people about the use of traditional medicine, conservation, and documentation mechanisms of traditional medicinal animals were gathered from all respondents [30]. Nature has been the source of medicinal agents for thousands of years and a number of modern drugs have been isolated from natural sources based on their use in traditional medicine [31].

The traditional knowledge of the use and conservation of the plants is still being transferred from generation to generation, but appeared to be aging. The problem of transfer of knowledge from the elders to the young generation probably arose following the introduction of modern education, religious, spiritual and culturerelated factors. Therefore, it is not only essential to conserve such a wealth of information hidden among the local people but also to apply modern science and technology to meet the ever increasing requirements of humankind. Furthermore, conservation of these biological resources is very important because their sustainable use can generate higher levels of employment and income. It was hypothesized that the findings may provide useful information for further scientific research to determine efficacies for documented ethnoproducts and practices to help improve animal health and human livelihood in Africa.

\section{Conclusion and Recommendations}

This study showed the wide use of medicinal plants and animals in Hawa Galan and Sayo District in meeting the primary healthcare needs of the community. A limited access to modern healthcare facilities could be considered as the main factors for the continuation of the traditional practice. There is an abundance of medicinal plants in the study area with almost all growth habitats which is probably attributed due to comfortable climate of the area. Even though there is a wealth of indigenous knowledge transfer is declining from generation to generation as a result of oral transmission. This finding therefore may imply that conducting indepth scientific studies may help elucidate the science underlying the efficacy of both animals and plants in managing health and this may lead to the discovery of useful Lead compound. There is a need therefore for the community to address the challenges of sustainable utilization and conservation of these medicinal organisms and resource ethnopractitioners.

\section{References}

1. Ngeh J Toyang, Jacob Wanyama, Mopoi Nuwanyakpa, Sali Djang (2007) Ethnoveterinary medicine, a practical approach to the treatment of cattle diseases in sub-Saharan Africa. Research Journal of Agricultural and Environmental Management 1(1).
2. Jarakabande Kaval (2002) ethnoveterinary medical traditions and methodology for theirocumentation, assessment and promotion. Foundation for Revitalisation of Local Health Traditions.

3. Blakeney M (1999) What is Traditional Knowledge? Why should it be protected? Who should protect it? For Whom? Understanding the Value Chain. UNESCO.

4. Mathias E, CM McCorkle (2004) Traditional livestock healers. Rev Sci Tech 23(1): 277-284.

5. Wanzala W, Zessin KH, Kyule NM, Baumann MPO, Mathias E, et al. (2005) Ethno veterinary medicine: A critical review of its evolution, perception, understanding and the way forward. Livestock Research for Rural Development 17(11): 1-31.

6. Dedeke GA, Soewu DA, Lawal OA, Ola M (2006) Pilot Survey of Ethnozoological Utilisation of Vertebrates in Southwestern Nigeria. Indilinga Afr J Indigenous Knowl Syst 5(1): 87-96.

7. Lev E (2003) Traditional healing with animals (zootherapy): medieval to present-day Levantine practice. J Ethno Pharmacol 85(1): 107-118.

8. Maria Costanza Torri (2013) Local Livelihoods and Human Health Care Traditional Veterinary in Rural Tamil Nadu. Journal of Developing Societies University of New Brunswick Canada.

9. Mathias E (2001) Introducing ethno veterinary medicine.

10. Costa Neto EM (2005) Animal based medicines: biological prospection and the sustainable use of zootherapeutic resources. Ann Acad Bras Cienc 77(1): 33-43

11. Adeola MO (1992) Importance of wild animals and their parts in the Culture, Religious Festivals, and Traditional Medicine, of Nigeria. Environmental Conservation.

12. A Diamandopoulos, DA Koutras, SG Marketos (1992) Healing rituals and sacred serpents. Lancet 340(8822): 223-225.

13. Tesfaye S, Kaleab Asres, Tsige Gebre Mariam (2004) Ethnobotanical and ethnopharmaceutical studies on medicinal plants of Chifra District, Afar Region, North Eastern Ethiopia. Ethiopian Pharmaceutical Journal 24(1): 41-58.

14. Alves RRN, Rosa IL (2005) Why study the use of animal products in traditional medicines? J Ethnobiol Ethnomed.

15. Yirga G (2010) Assessment of indigenous knowledge of medicinal plants in Central Zone of Tigray, Northern Ethiopia. Afr J Plant Sci 4(1): 6-11.

16. Zerabruk S, Yirga G (2011) Traditional knowledge of medicinal plants in Gindeberet District, Western Ethiopia. South Afr J Bot.

17. Amenu F (2007) Use and management of medicinal plants by indigenous people of Ejaji area (chelya woreda) west shoa, Ethiopia: An ethnobotanical approach. Addis Ababa University.

18. Yumoto T, Yamagiwa J, Mwanza N, Maruhashi T (1994) List of plant species identified in Kahuzi-Biega National Park, Zaire. Tropics 3(3+4): 295-308.

19. Lengkeek G (2003) Diversity makes a difference. Farmers managing inter- and intraspecific tree species diversity in Meru, Kenya. Wageningen University and Research Centre.

20. Abebe D, Ayehu A (1993) Medicinal Plants and Enigmatic Health Practices of Northern Ethiopia. Addis Ababa Ethiopia.

21. Souto WMS, Mourão JS, Barboza RRD, Alves RRN (2011) Parallels between zootherapeutic practices in ethnoveterinary and human complementary medicine in northeastern Brazil. J Ethnopharmacol 134(3): 753-767.

22. Kokwaro OJ (2009) Medicinal plants of East Africa. $3^{\text {rd }}$ Edition.

23. Diriba Gurmesa, Debela Abdeta (2019) Identification of Poisonous Plants and Their Toxics Effects on Livestock in Horo Buluk District, Horo 
Guduru Wollega Zone, Oromia Regional State, Western Ethiopia. Biomed J Sci \& Tech Res 23(3): 17391-17395.

24. Wanzala W, Takken W, Mukabana WR, Pala AO, Hassanali A (2012) Ethnoknowledge of Bukusu community on livestock tick prevention and control in Bungoma district, western Kenya. J Ethnopharmacol 140(2): 298-324.

25. Kelbessa E, Demissew S, Woldu Z, Edwards S (2000) Some threatened Endemic plants of Ethiopia. In The status of some plants in parts of tropical Africa. East and Central Africa NAPRECA 1992: 35-55.

26. Cunningham A (2001) Applied Ethnobotany: People, Wild Plant Use and Conservation.

27. Haileselasie T (2012) Traditional zootherapeutic studies in Degu'a Tembien, Northern Ethiopia. Curr Res J Biol Sci 4(5): 563-569.

ISSN: 2574-1241

DOI: $10.26717 /$ BJSTR.2020.28.004620

Debela Abdeta. Biomed J Sci \& Tech Res

CC (P) This work is licensed under Creative

Submission Link: https://biomedres.us/submit-manuscript.php
28. Yirga G, Teferi M, Gebreslassea Y (2011) Ethnozoological study of traditionalmedicinal animals used by the people of Kafta Humera District, Northern Ethiopia. Int J Med Med Sci 3(10): 316-320.

29. Borah MP, Brasad SB (2017) Ethnozoological study of animals-based medicines used by traditional healers and indigenous inhabitants in the anointing area Gibbon Wildlife Sanctuary, Assam, India. J Ethnobiol Ethnomed. 13: 39.

30. Kendie FC, Mekuriaw SA, Dagnew MA (2018) Ethnozoological study of traditional medicinal appreciation of animals and their products among the indigenous people of Metema Woreda North-Western Ethiopia. Journal of Ethnobiology and Ethnomedicine 14(1).

31. Guluma K, Debela A, Morka A (2017) Ethnoknowledge of plants used in veterinary practices in Midakegn district, west showa of Oromia region Ethiopia. Journal of Medicinal Plants Studies 5(5): 282-288.

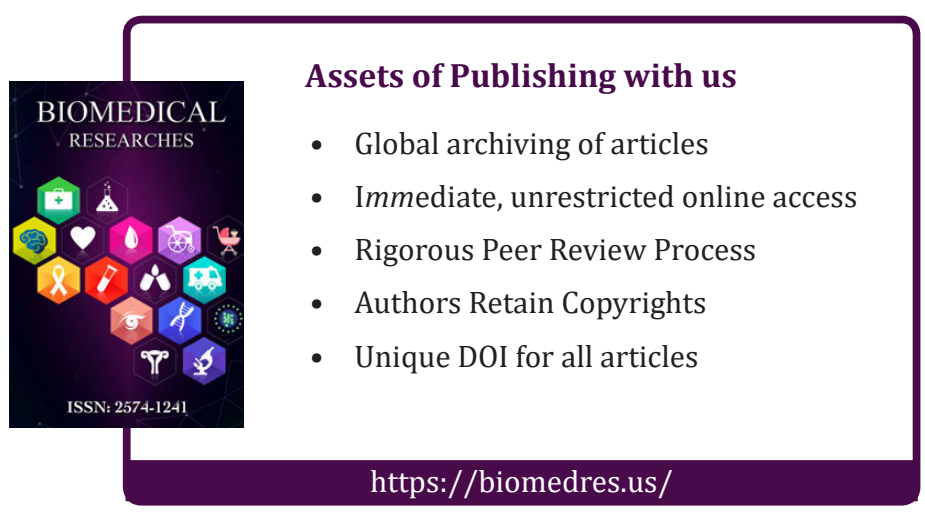

